\title{
CARF (collaborator of ARF) interacts with HDM2: Evidence for a novel regulatory feedback regulation of CARF-p53-HDM2-p21WAF1 pathway
}

\author{
M. KAMRUL HASAN ${ }^{1,3}$, TOMOKO YAGUCHI ${ }^{1}$, JUN ITADANI HARADA ${ }^{1}$, \\ TAKASHI HIRANO ${ }^{1}$, RENU WADHWA $^{1,2}$ and SUNIL C. KAUL ${ }^{1}$ \\ ${ }^{1}$ National Institute of Advanced Industrial Science and Technology, 1-1-1 Higashi, \\ Tsukuba, Ibaraki 305-8562; ${ }^{2}$ Department of Chemistry and Biotechnology, \\ School of Engineering, University of Tokyo, Hongo, Tokyo 113-8656, Japan
}

Received September 24, 2007; Accepted November 28, 2007

\begin{abstract}
We initially cloned CARF (collaborator of ARF), as a novel ARF-binding protein by a yeast interaction screen. It also interacts with p53 directly leading to ARF-independent enhancement of $\mathrm{p} 53$ function and in turn undergoes a negative feedback regulation. Herein we report that i) CARF interacts with HDM2 and undergoes degradation by an HDM2dependent proteasome pathway, and ii) it acts as a transcriptional repressor of HDM2. By overexpression and silencing studies, we demonstrated that CARF exerts a vital control on the p53-HDM2-p21WAF1 pathway that is frequently altered in cancer cells.
\end{abstract}

\section{Introduction}

The development of human cancers is frequently associated with the disruption of the two major tumor suppressor pathways governed by activities of p53 and retinoblastoma (pRB) proteins that are also closely involved in execution of cellular senescence (1-9). The INK4a/ARF locus encodes two distinct tumor suppressors, a member of the INK4 cyclin-dependent kinase inhibitor family, p16 ${ }^{\text {INK4A }}$, and a completely unrelated protein, ARF (p19ARF in mouse and p14ARF in human), encoded by an alternative reading frame. These two INK4A-encoded proteins regulate cell cycle progression via the $\mathrm{pRB}$ and $\mathrm{p} 53$ pathways (10-15). ARF

Correspondence to: Dr Sunil C. Kaul, National Institute of Advanced Industrial Science and Technology (AIST), Central 4, 1-1-1 Higashi, Tsukuba, Ibaraki 305-8562, Japan

E-mail: s-kaul@aist.go.jp

Present address: ${ }^{3}$ Tokyo Women's Medical University, Tokyo, Japan

Key words: $\mathrm{p} 14^{\mathrm{ARF}(a l t e r n a t i v e ~ r e a d i n g ~ f r a m e) ~}$, collaborator of ARF, $\mathrm{p} 53$, HDM2, interaction, RNA interference, feedback regulation, transcriptional repressor has been shown to affect both p53- and RB-mediated cell cycle arrest and apoptosis and thus, play a key role in tumor suppressor mechanisms (16-18). It has been shown to bind to MDM2 (mouse double minute 2, called HDM2 in humans), sequester it within the nucleoli, promote its degradation and inhibit E3 ubiquitin ligase activity that is required for p53 and pRB degradation (19-27). Although ARF-HDM2 binding in the nucleolus was initially identified as a key factor in regulation of $\mathrm{p} 53$ and $\mathrm{pRB}$ activities, a more complex picture emerged when certain studies showed that nucleolar localization of ARF was not essential although it may enhance the availability of ARF to inhibit HDM2 $(28,29)$. Furthermore, the function of ARF did not fully correlate with MDM2 import to the nucleolus suggesting that ARF function requires other events (29) perhaps mediated by its binding partners including E2F family members, spinophilin, topoisomerase I, MdmX, Pex19p, CARF, Cyclin G1, p120 (E4F) and its homooligomers (30-39).

We have previously cloned CARF as an ARF-binding protein by yeast two hybrid screening (40). It was localized in the human chromosome $4 \mathrm{q} 35$, a region associated with esophageal adenocarcinoma $(40,41)$. CARF was found to be expressed in most human tissue and was able to activate ARF-dependent p53 function $(36,40)$. In the absence of ARF, CARF directly binds to p53 causing its stabilization and functional activation, and in turn undergoes negative feedback regulation (42). In the present study, we addressed the molecular mechanism of CARF regulation and found that it is ubiquitinated and negatively regulated by p53 and HDM2 by proteasome- dependent degradation. To protect itself from degradation, CARF acts as a transcriptional repressor of HDM2, nullifies the antagonist effect on p53 and activate ARF-p53-HDM2-p21WAF1.

\section{Materials and methods}

Plasmids, cells, transfections and infection. CARF was cloned into mammalian expression plasmid pcDNA3.1/V5 (Invitrogen) pMT-vector and bacterial expression plasmid pQE30 (Qiagen) to encode V5, Zn inducible CARF-myc and His-tagged proteins, respectively. Full-length HDM2 and its 
deletion mutants were cloned into pcDNA3.1/V5 to obtain V5-tagged HDM2 in human cells. HDM2 promoter-driven YFP expression was obtained by transfecting the cells with pU293 plasmid (kindly provided by Dr Uri Alon, Israel). Plasmid containing luciferase driven by HDM2 promoter was a kind gift from Bert Vogelstein, USA. pCMVHA-Ub encoding HA-Ub was provided by Dr Koyomi Miyazaki (AIST, Tsukuba, Japan). shRNA plasmid for HDM2 was prepared as described (43-45). Target sequences used for HDM2-shRNA plasmids were GCCATTGCTTTTGAA GTT and GAAGCAGTAGCAGTGAATC, respectively. Human normal (TIG-1, lung fibroblast) and cancer (U2OS, osteosarcoma, HeLa, cervical carcinoma, HepG2, hepatocyte cell line, HCT116, colon carcinoma; kindly provided by B. Vogelstein, USA) cells were cultured in Dulbecco's modified Eagle's minimal essential medium supplemented with $10 \%$ fetal bovine serum. Transfections were performed using Lipofectamine ${ }^{\mathrm{TM}}$ Plus (Life Technologies, Inc.). Plasmid DNA (3 and $10 \mu \mathrm{g}$ ) was used per $80 \%$ confluent $6-$ and $10-\mathrm{cm}$ dish, respectively. For stable transfections, pPur plasmid was co-transfected followed by selection in puromycin $(2-3 \mu \mathrm{g} / \mathrm{ml})$ supplemented medium. Several colonies were picked up by ring isolation. Cells were expanded and the expression of the transfected cDNAs or the target protein (in case of siRNA transfections) was analyzed by Western blotting as described below.

In vivo co-immunoprecipitation and immunodepletion. Cell lysates (400 $\mu \mathrm{g}$ protein) in $400 \mu \mathrm{l}$ Nonidet P-40 lysis buffer were incubated at $4{ }^{\circ} \mathrm{C}$ overnight with an antibody used for immunoprecipitation. Immunocomplexes were separated by incubation with Protein-A/G Sepharose at $4^{\circ} \mathrm{C}$ for $30 \mathrm{~min}$, and Western blotting was performed with the indicated antibodies as described below. For immunodepletion, lysates were immunoprecipitated with the indicated antibody for three rounds of immunoprecipitation as described above. The amount of CARF left in the lysate after each round of immunoprecipitation was examined by Western blotting as described below.

In vitro and in vivo CARF and HDM2 interaction assay. For in vitro interactions, $125 \mathrm{ng}$ of bacterially expressed His-CARF was incubated with an equal amount of GSTHDM2 and $250 \mathrm{ng}$ of bovine serum albumin (BSA) in NP-40 lysis buffer. After incubation at $4^{\circ} \mathrm{C}$ for $4 \mathrm{~h}$, His-CARF was pulled-down with Ni-NTA agarose beads $(20 \mu 1)$. After six washes with NP-40 lysis buffer, the pellet was boiled in SDS sample buffer. The eluted proteins were electrophoresed through a $10 \%$ polyacrylamide gel, transferred onto a nylon membrane and blotted with anti-HDM2 and anti-His antibodies to detect HDM2 and CARF, respectively. For in vivo interactions, HepG2 cells were transfected with expression plasmids encoding V5-tagged HDM2 and its deletion mutants encoding $\mathrm{N}$-terminus (N1, 204 amino acids and N2, 435 amino acids) and C-terminus (C2, 220 amino acids). HDM2 protein was immunoprecipitated with anti-V5 antibody and the co-immunoprecipitating CARF was examined by Western blotting with anti-CARF antibody. Immunoprecipitated HDM2-V5 proteins were examined by Western blotting with anti-V5 antibody.
In vivo ubiquitylation of CARF. U2OS cells were transfected with expression plasmids encoding CARF-V5, HA-Ub and HDM2 as indicated. After $48 \mathrm{~h}$ of transfection, cell lysates were made under denaturing conditions as described earlier (34). CARF-V5 was immunoprecipitated with anti-V5 antibody and was detected by Western blotting with anti-V5 (Invitrogen) and anti-HA (Santa Cruz Biotech) antibodies.

Western analysis. The protein sample (10-20 $\mu \mathrm{g})$ separated on a SDS-polyacrylamide gel was electroblotted onto a nylon membrane (Millipore) using a semidry transfer blotter (Biometra, Tokyo). Immunoblotting was performed with anti-HA tag (Santa Cruz), anti-V5 tag (Invitrogen), anti-p53 (DO-1), anti-p21 (C-19), anti-MDM2 (SMP-14, Santa Cruz) anti-BAX (BD Transduction), anti-PUMA (ProScience), anti-actin (Boehringer Mannheim) and anti-CARF $(40,42)$ antibodies. The immunocomplexes formed were visualized with horseradish peroxidase (HRP)-conjugated rabbit antimouse immunoglobulin $\mathrm{G}(\mathrm{IgG})$ antibody (ECL kit, Amersham Pharmacia Biotech).

Immunostaining. Cells grown on glass coverslips placed in $35-\mathrm{mm}$ plastic dishes were washed with cold phosphatebuffered saline (PBS) and fixed with $4 \%$ formaldehyde for $10 \mathrm{~min}$ at room temperature. Fixed cells were washed with PBS and permeabilized with $0.2 \%$ Triton-X100 in PBS for $20 \mathrm{~min}$. Cells were then incubated in blocking buffer $(2 \%$ BSA) containing the primary antibody (either tag-specific or protein-specific as indicated in the legends of Figs. 1 an 3) for $1 \mathrm{~h}$ and washed extensively in PBS before incubation with the appropriate fluorochrome-conjugated secondary antibody for another $30 \mathrm{~min}$. Secondary antibodies used were Alexa-488-conjugated goat anti-rabbit and anti-mouse or Alexa-594-conjugated goat anti-rabbit and anti-mouse (Molecular Probes). After six washes in PBS with $0.1 \%$ Triton X-100, cells were overlaid with a coverslip with fluoromount (Difco). The cells were examined on a Carl Zeiss microscope attached with Photomerics Synsys monochrome charge-coupled device (CCD) or on an Olympus microscope equipped with a CSU 20 spinning-disk confocal scanner (Yokogawa Electric Corporation) and ultra-high-sensitive HARP camera (Hitachi Kokusai Electric, Japan) and CSU-20 scanner (Yokogawa, Japan). The extent to which the two proteins co-localized was assessed by combining the images using Metamorph software.

CARF SiRNA. 21-Nucelotide RNAs were chemically synthesized using Expedite RNA phosphoramidites and thymidine phosphoramidite. Synthetic oligonucleotides were deprotected and gel purified. Sequences of two control and two target oligos for CARF were: 5'-AAGACCGAGUCCA UGAGGCUT-3', 5'-GCCUCAUGGACUCGGUCUUUT-3' and 5'-CGGAGUACCUGAGCCAGAAUT-3', 5'-UUCUGG CUCAGGUACUCCGUT-3', respectively. For annealing of siRNAs, $20 \mu \mathrm{M}$ of two control or target single strands were incubated in annealing buffer $(100 \mathrm{mM}$ potassium acetate, $30 \mathrm{mM}$ HEPES-KOH at $\mathrm{pH}$ 7.4, $2 \mathrm{mM}$ magnesium acetate) for $1 \mathrm{~min}$ at $90^{\circ} \mathrm{C}$ followed by $1 \mathrm{~h}$ at $37^{\circ} \mathrm{C}$. Transfections of siRNA duplexes were carried out using Oligofectamine reagent (Life Technologies). Of the $20 \mu \mathrm{M}$ duplexes, 1-5 $\mu 1$ were 
used per 12-well dish and were assayed after 24-48 h by immunostaining and Western blotting with anti-CARF antibody.

$R T-P C R$. Total RNA was isolated using TRIzol reagent (Invitrogen) and $2 \mu \mathrm{g}$ was used to amplify $695 \mathrm{bp}$ HDM2 fragment using HDM2 specific primers (sense 5'-GAGATA TGTTGTGAAAGA-3' and antisense 5'-AATGGCATT AAGGGGCAAACT-3') for 28,33 and 38 cycles $\left(94^{\circ} \mathrm{C}\right.$ for $5 \mathrm{~min} ; 94^{\circ} \mathrm{C}$ for $1 \mathrm{~min} ; 48^{\circ} \mathrm{C}$ for $1 \mathrm{~min}$ and $72^{\circ} \mathrm{C}$ for $1 \mathrm{~min}$ ). Actin was used as an internal control and was amplified using actin-specific primers (sense 5'-CTGGAACGGTGAAG GTGACA-3' and antisense 5'-CTAAGGGACTTCCTGT AACAATGCA-3'). The amplified product was resolved through $1 \%$ agarose and visualized using ethidium bromide.

Reporter assays. For HDM2 promoter assay, U20S cells were transfected with pHDM2-luc, pMTCARF-myc and pPur plasmids and the stably transfected cells were selected by puromycin $(2.5 \mu \mathrm{g} / \mathrm{ml})$. Cells $\left(1 \times 10^{4}\right.$ cells/well $)$ were plated in a 24-well plate and transfected with control or CARF siRNA as described above. Sixty hours posttransfection, $100 \mu \mathrm{M} \mathrm{ZnSO}_{4}$ was added to induce the expression of exogenous CARF-myc and the cells were assayed for luciferase $24 \mathrm{~h}$ later using luciferase assay kit (Promega). For p53 reporter assay, HCT116 cells $\left(1 \times 10^{4}\right.$ cells/well) grown in a 24-well plate were transfected with CARF specific or control siRNA along with pWWP-Luc (p21 promoter reporter construct). Cells were lysed after $48 \mathrm{~h}$ and quantitated for luciferase expression using luciferase assay reagent (Roche) and a luminometer (Wallac multi-label counter).

\section{Results and Discussion}

In light of our previous findings that CARF undergoes proteasome-mediated degradation along with p53 (42), it was anticipated that HDM2 might be involved in this process. Therefore, we examined if CARF interacts with HDM2 by performing in vitro and in vivo co-immunoprecipitation assays. As shown in Fig. 1a, HDM2 was co-immunoprecipitated with CARF from HeLa cell lysates. Co-precipitation of CARF was also observed in HDM2-immunocomplexes (data not shown). We also examined if the three proteins (CARF, HDM2 and ARF) form a complex. U2OS cells (that lack endogenous p14ARF) were transfected with expression plasmids encoding CARF-V5, p14ARF-myc and HDM2 proteins. Anti-CARF, anti-p14ARF and anti-HDM2 antibodies were used to immunodeplete the respective proteins from the lysates by three rounds of immunoprecipitation. The remaining amount of CARF-V5 left in each case was examined by Western blotting with anti-V5 antibody. As shown in Fig. 1b, CARF-V5 was depleted from the lysates not only as a consequence of CARF immunoprecipitation, but also by p14ARF and HDM2 immunoprecipitations. These data suggested that CARF interacts with p14ARF (36) and HDM2 in cells.

To further confirm the CARF-HDM2 interactions, we performed the in vitro pull down assays. Bacterially expressed His-CARF ( 85 kDa) and GST-HDM2 ( 115 kDa) proteins were mixed in a tube and His-CARF was pulled down with Ni-NTA agarose. Co-pulldown of GST-HDM2 was examined by Western blotting with anti-HDM2 antibody. As shown in Fig. 1c, GST-HDM2 was pulled down along with His-CARF (Fig. 1c, lane 4). Ni-NTA, by itself, did not cause any pull down of GST-HDM2 (Fig. 1c, lane 2). In parallel experiments using equal amounts of GST-p53 and GST-HDM2, pull down of GST-HDM2 was higher than GST-p53 (42) and data not shown. These data demonstrated that i) the interaction of CARF and HDM2 is specific and is not due to the binding of GST to His-CARF, ii) CARF may bind to HDM2 more strongly than to p53, and iii) CARF and HDM2 can bind directly and do not need other proteins. Consistent with these biochemical data on interactions of CARF and HDM2 in vivo and in vitro, we detected significant co-localization of the proteins in the nucleus (seen as yellow color in the merged photomicrographs) by fluorescence microscopy (Fig. 1d). The data were further strengthened by in vivo co-immunoprecipitation of HDM2 and its deletion mutants with CARF. We found that CARF and its N-terminus deletion mutants (N1 and N2) could be immunoprecipitated with full-length. However, it did not precipitate with C-terminus mutant (C2) that lacked N-terminal 125 amino acids (Fig. 1e). Of note, although the amount of immunoprecipitated $\mathrm{C} 2$ was manyfold more than the N1 and N2 (Fig. 1e, lower panel), no immunoprecipitation of CARF was obtained. The data defined that the N-terminal region of HDM2 is essential for binding to CARF. Although HDM2 was able to bind to CARF directly in vitro (Fig. 1c), the possibility that the CARF-p53 complexes (42) may be recruited to the $\mathrm{N}$-terminus of HDM2 in vivo cannot be ruled out.

CARF-HDM2 binding as shown in Fig. 1 suggested that HDM2 might be involved in degradation of CARF by a proteasome-mediated pathway. We further investigated whether CARF undergoes ubiquitylation. Cells were transfected with expression plasmids encoding CARF-V5, HDM2 and HA-tagged ubiquitin and were examined for levels of CARF and CARF-V5 expression and their ubiquitination by Western blotting and immunoprecipitation by anti-CARF, V5 and HA antibodies as shown in Fig. 2. We found: i) CARF and CARF-V5 showed a decrease when ubiquitin was overexpressed (Fig. 2a), ii) CARF-V5 was immunoprecipitated with anti-V5 antibody and detected by Western blotting with either anti-V5 or anti-HA antibody. Ubiquitinated CARF was detected with anti-HA antibody (Fig. 2a). As expected, ubiquitinated CARF-V5 appeared as a smear, that reacted more strongly with anti-HA antibody than with the anti-V5 antibody. Furthermore, the level of CARF was found to decrease with overexpression of HDM2 suggesting its enhanced degradation (Fig. 2a and b). The data were also supported by a several-fold increase in the amount of ubiquitylated CARF with the treatment of cells with MG132 (Fig. 2a). Of note, over-expression of HDM2 not only caused a reduction in CARF expression, but also in its downstream effectors, p53 and p21 (Fig. 2b). In agreement with these findings, silencing of HDM2 by shRNA in U2OS cells resulted in an increased level of CARF in cells (Fig. 2c). These data clearly showed that CARF undergoes HDM2mediated ubiquitination and degradation by a proteasome pathway. Taken together with the data in Fig. 1, we found 
a

c

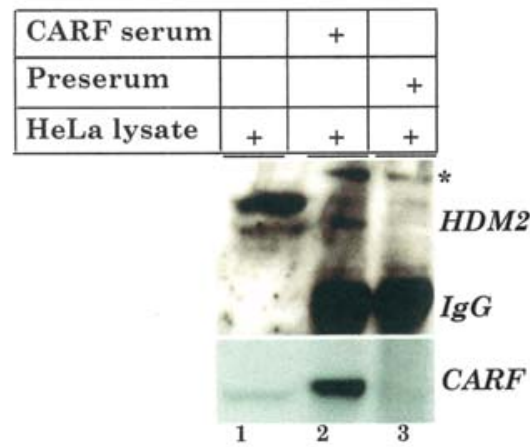

b
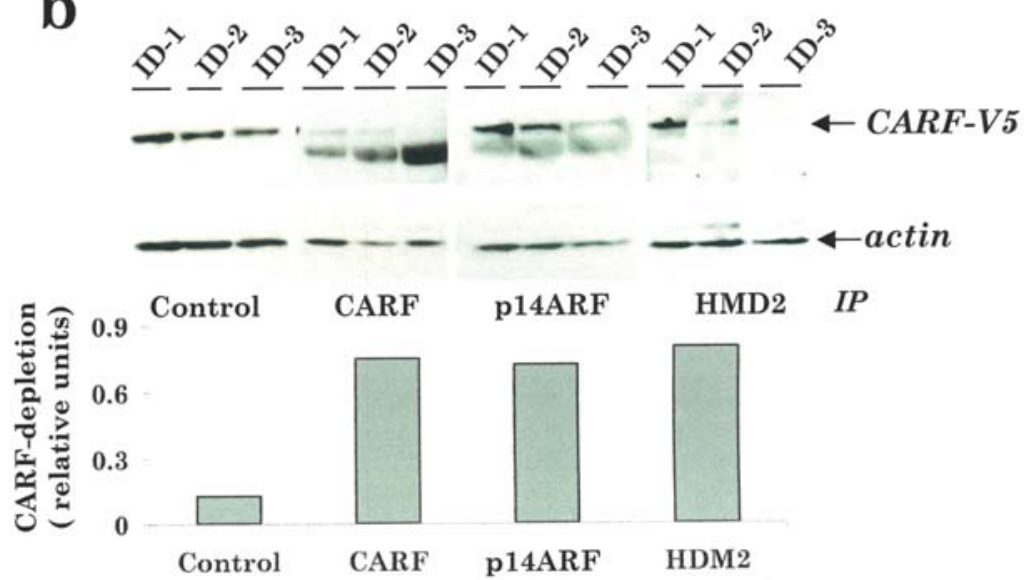

d

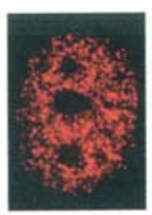

CARF

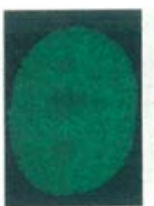

HDM2 Overlay

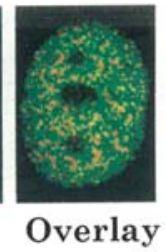

$\mathbf{e}$

\begin{tabular}{|c|c|c|c|}
\hline p53 BD & $\begin{array}{l}\text { Acidic Zinc } \\
\text { domain finger } \\
221\end{array}$ & \multirow{2}{*}{\multicolumn{2}{|c|}{$\begin{array}{l}\frac{\text { RING finger }}{438} \\
\infty_{478}^{48}{ }_{478 a}\end{array}$}} \\
\hline 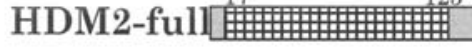 & IIIIII & & \\
\hline N1 嘈典典典典 & & & 204aa \\
\hline N2 & HHI & & $435 a$ \\
\hline $\mathrm{C} 2$ & 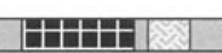 & 8 & $349 a$ \\
\hline
\end{tabular}

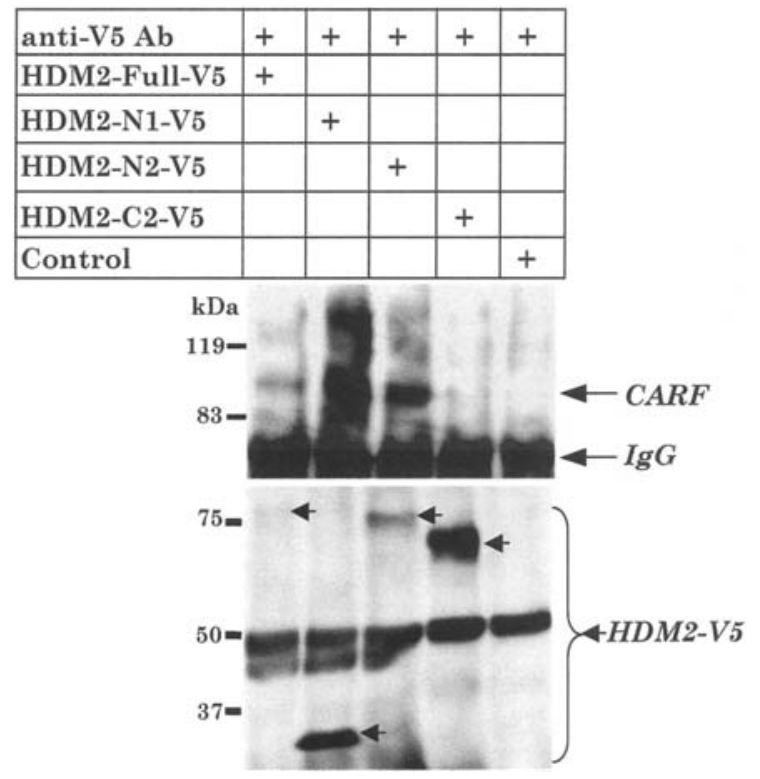

Figure 1. CARF interacts with HDM2 in vivo and in vitro. (a) In vivo co-immunoprecipitation of CARF and HDM2. Anti-CARF antibody was added in HeLa cell lysates $(200 \mu \mathrm{g})$ and pulled down by Protein-A agarose beads. CARF immunocomplexes were analyzed for the presence of HDM2 by Western blotting with anti-MDM2 antibody (SMP-14). HDM2 co-immunoprecipitated with CARF (lane 2) but not with control antibody (lane 3). Lane 1 shows HDM2 and CARF input signals in $10 \mu \mathrm{g}$ of cell lysates. A high molecular weight protein that cross-reacted with both pre- and CARFserum is shown by asterisk. (b) U2OS cells were transfected with expression plasmids encoding CARF-V5, p14ARF-myc and HDM2. Cell lysates were immunodepleted for CARF, p14ARF and HDM2 by three rounds of immunoprecipitation with respective antibodies. The amount of CARF-V5 remained in the lysates after each round of immunodepletion was examined by Western blotting with anti-V5 antibody. The amount of CARF depleted from the 1st to 3rd round of immunoprecipitation with each antibody was quantitated and plotted. As seen, anti-CARF, anti-p14ARF and anti-HDM2 (but not the control) antibodies caused immunodepletion of CARF suggesting that it interacts with $\mathrm{p} 14 \mathrm{ARF}$ and HDM2 in cells. (c) In vitro co-precipitation of CARF and HDM2. His-CARF ( 85 kDa) and GSTHDM2 $(\sim 115 \mathrm{kDa})$ were mixed in the presence of BSA to avoid unspecific aggregation of the two proteins. His-CARF was pulled down from the mixture by Ni-NTA agarose beads. Co-precipitation of GST-HDM2 was detected by Western blotting with anti-HDM2 antibody (lane 4). GSTHDM2 was pulled down by CARF (lane 4) but not with Ni-NTA beads (lane 2). Higher molecular weight band marked by asterisks in lane 4 represents an artifact. (d) Co-immunostaining of CARF and HDM2 in the nucleus of U2OS cells. Significant overlap of CARF and HDM2 staining is seen in the nucleoplasm. (e) Schematic diagram showing HDM2 mutants used for co-immunoprecipitation with CARF. Cells were transfected with expression plasmids encoding HDM2-V5 proteins that were immunoprecipitated with anti-V5 antibody. Co-precipitation of CARF was examined with Western blotting with anti-CARF antibody. Immunoprecipiation of HDM2-V5 proteins was confirmed with Western blotting with anti-V5 antibody. Whereas the full-length and N-terminal regions (N1 and N2) precipitated CARF, C2 did not. The result implied that the N-terminal region (amino acid residues 17-125) is required for its interactions with CARF. 
a

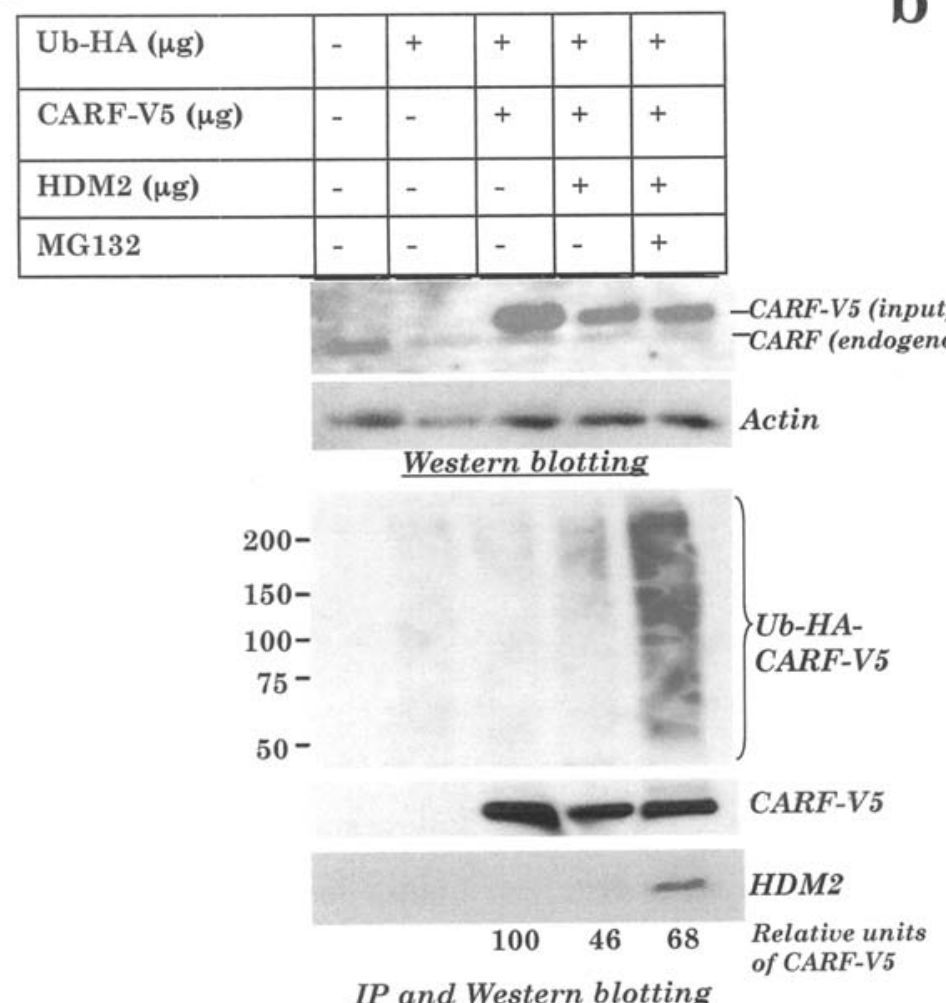

b

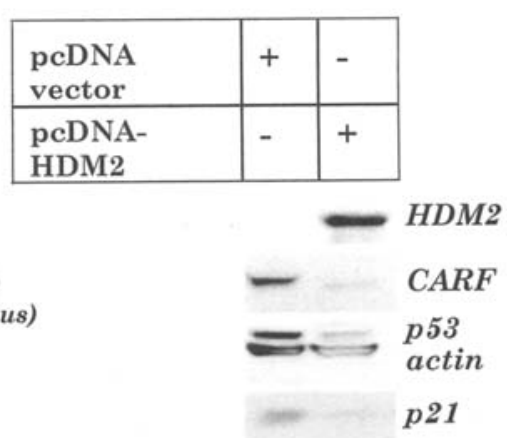

C

\begin{tabular}{|l|l|c|}
\hline $\begin{array}{l}\text { HDM2- } \\
\text { shRNA }\end{array}$ & - & + \\
\hline Vector & + & - \\
\hline
\end{tabular}

HDM2

CARF

actin
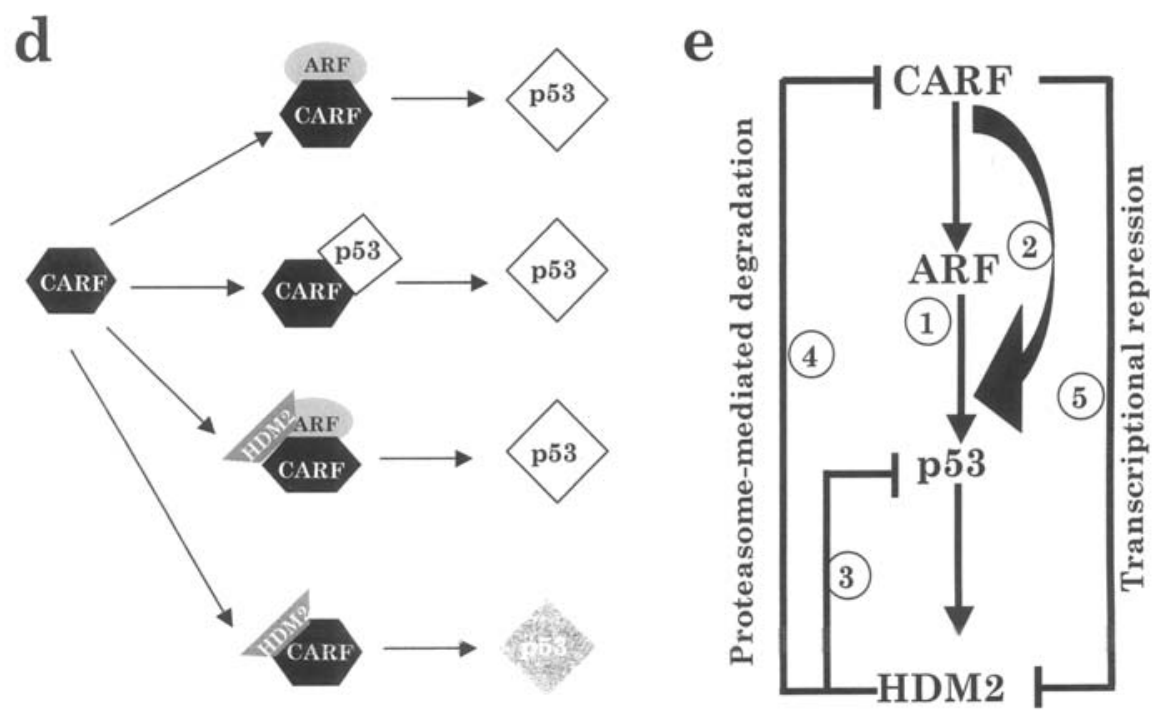

Figure 2. CARF is downregulated by HDM2 by proteasome-mediated degradation. (a) U2OS cells were transfected with expression plasmids as indicated. Level of CARF and CARF-V5 was examined by Western blotting with anti-CARF and anti-V5 antibodies (upper panel). CARF-V5 was immunoprecipitated by anti-V5 antibody and immunocomplexes were examined for ubiquitinated CARF (high molecular weight bands) by Western blotting with anti-HA antibody. CARF was detected by anti-HA antibody supporting its ubiquitination by Ub-HA. The ubiquitinated CARF (appeared like high molecular weight smear) was increased when proteasome-mediated degradation was blocked by MG132 (lower panel). (b) Cells were transfected with expression plasmids as indicated. Western blotting for HDM2, CARF, p53 and p21WAF1 revealed that the overexpression of HDM2 resulted in decreased level of CARF protein. This was also accompanied by a decrease in the level of p53 and p21WAF1. Actin was used as a loading control. (c) Cells were transfected with shRNA plasmids for HDM2. Silencing of HDM2 resulted in the increased amount of CARF in cells as seen by Western blotting. Actin was used as an internal loading control. (d) Diagram showing binding partners of CARF. It interacts with ARF, p53 and HDM2. Activated p53 and degraded p53 is shown by white and grey diamonds, respectively. Whereas HDM2-CARF complexes are directed for proteasome degradation, ARF-CARF-HDM2 complexes may be stable in the nucleolus. (e) Diagram showing activation of p53 function by CARF in ARF-dependent and -independent manners (1 and 2). Negative feedback regulation of CARF by HDM2-mediated degradation is similar to direct HDM2 feedback regulation of p53 itself ( 3 and 4). Transcriptional repression function of CARF for HDM2 (5).

that CARF (that was previously shown to upregulate p53 function in an ARF-dependent and -independent manner) interacts with HDM2 and gets degraded by an HDM2- mediated proteasome degradation pathway (Fig. $2 \mathrm{~d}$ and e). Whereas p53 is upregulated by CARF, HDM2 (p53 downstream effector and antagonist) degrades CARF in a 
a

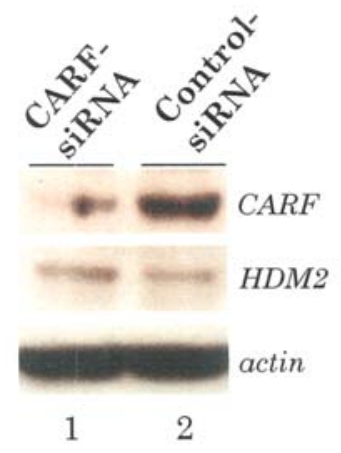

C
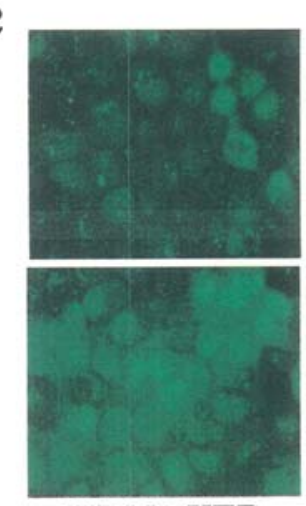

HDM2-YFP
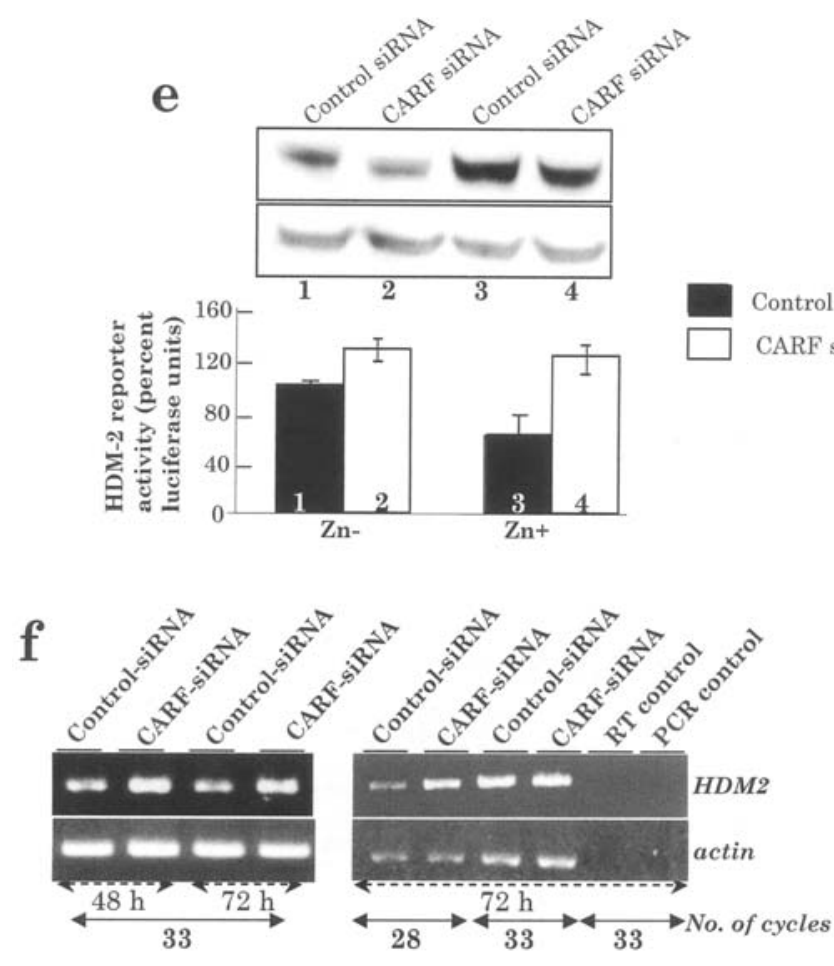

b

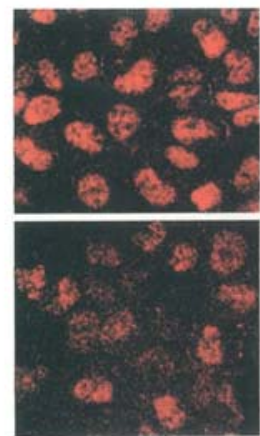

CARF

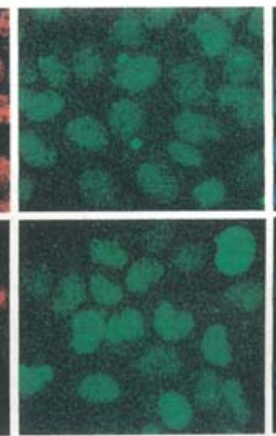

HDM2

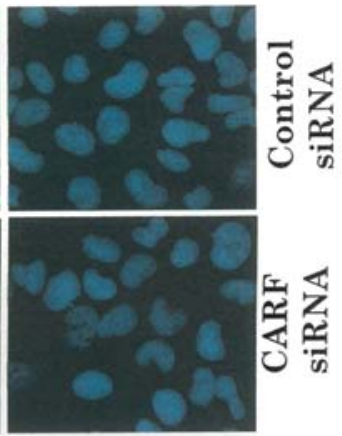

DAPI d

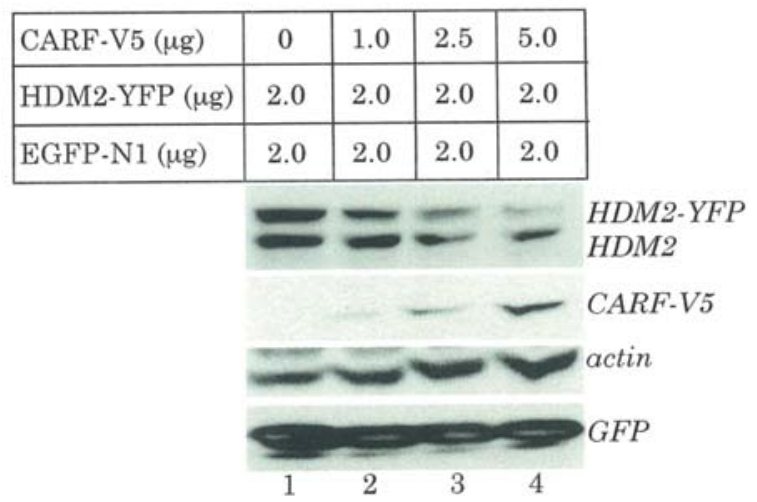

Figure 3. CARF is a transcriptional repressor of HDM2 in vivo. (a) U2OS cells were transfected with control (vector only) and target (CARF-specific) siRNA. Cell lysates were made $48 \mathrm{~h}$ after transfection and Western blotting was carried out for CARF, HDM2 and actin. The amount of CARF was reduced to $>70 \%$ in CARF-siRNA transfected cells. Cells knocked-down for CARF showed higher amounts of HDM2 (compare lane 1 and lane 2). Actin was used as an internal control. (b) CARF siRNA-transfected cells were examined for HDM2 by immunostaining. CARF-compromised cells showed a higher level of HDM2 expression. (c) U2OS cells were co-transfected with pU293 (HDM2-YFP driven by native HDM2-promoter). Cells with stable

Control siRNA expression of the HDM2-YFP reporter were grown on glass coverslips and CARF siRNA transfected with CARF-specific and control siRNA. After $48 \mathrm{~h}$ of transfection, cells were fixed and observed under a microscope. CARF-targeted cells show a higher level of HDM2-YFP reporter. (d) Cells were transfected with HDM2 promoter-driven HDM2-YFP reporter and increasing amounts of CARF-V5 expressing plasmids. Increase in CARF-V5 expression resulted in corresponding decrease in endogenous HDM2 and exogenous HDM2 (HDM2-YFP reporter). Actin was used as an internal loading control and GFP as transfection efficiency control. (e) Cells stably transfected with zinc inducible CARF and HDM2 promoter-driven luciferase reporter plasmid were transfected with CARF siRNA. Luciferase activity was quantitated as described in Materials and methods. CARF overexpression resulted in reduced HDM2 promoter activity and CARF targeting with siRNA abrogated this effect demonstrating that CARF acts as a transcriptional repressor of HDM2. (f) RNA was isolated from CARF-siRNA transfected U2OS cells at 48 and $72 \mathrm{~h}$ after transfection and RT-PCR was performed for HDM2 and actin (a loading control). The mRNA level of HDM2 was increased in CARF targeted cells (lane 2 and 4 from the left) both at 48 and $72 \mathrm{~h}$ of tranfection. PCR amplification for 28 and 33 cycles revealed clear differences in the amount of HDM2 transcript in control and CARF siRNA transfected cells. negative feedback regulatory loop (Fig. 2e). Degradation of CARF by HDM2 thus provides a mechanism by which this activator of p53 can be downregulated, allowing cells to progress through the cell cycle.
To understand the functional consequences of CARFHDM2 interactions, we employed siRNA-mediated knockdown of CARF in U2OS cells. As shown in Fig. 3a, we found that the knockdown of CARF by CARF-specific siRNA caused 

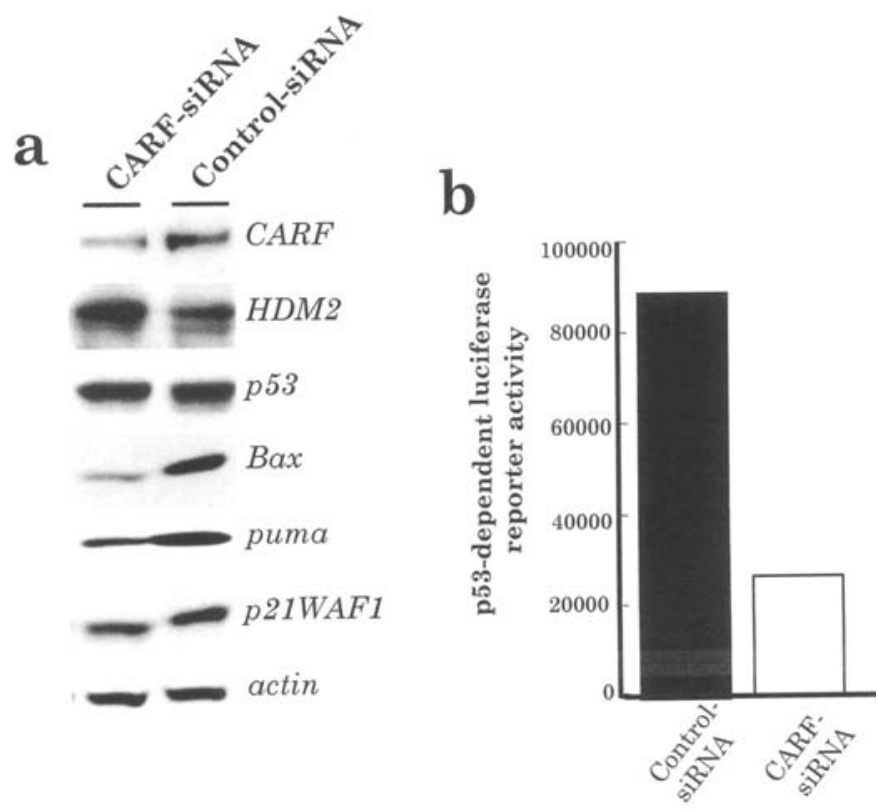

Figure 4. CARF regulates p53-HDM2-p21WAF1 pathway. (a) U2OS cells were transfected with CARF-siRNA and examined for p53 downstream effectors by Western blotting with specific antibodies. Whereas the level of HDM2 expression was increased, the level of p21WAF1, BAX and PUMA showed a significant decrease. (b) HCT116 cells were transfected with CARF-siRNA and a p53-dependent reporter plasmid, pWWP-Luc. Luciferase activity in an equal number of cells was assayed at $48 \mathrm{~h}$ posttransfection. The p53-dependent luciferase reporter activity was reduced in CARF-targeted cells.

an upregulation of HDM2 as observed by Western blotting as well as immunostaining using CARF and HDM2 specific antibodies (Fig. 3a and b). The data raised two new possibilities: i) CARF is essential for the proteasome degradation pathway and thus knockdown by siRNA resulted in an accumulation of HDM2 that itself gets degraded by the proteasome pathway and ii) CARF may act as a transcriptional repressor of HDM2. Based on the predominant nuclear localization of CARF and its serine-rich structure, we anticipated that it might have a role as a regulator of transcription. Hence, we performed two different assays. Cells stably expressing HDM2 promoter (3.5 kb 5' UTR containing P1 and P2 HDM2 promoter regions)-driven HDM2-YFP reporter (pU293) were made and expression of the HDM2-YFP reporter was examined following CARF-siRNA transfections. As shown in Fig. 3c, YFP reporter was highly expressed in CARF-siRNA transfected cells suggesting that normally CARF downregulates HDM2 and thus knockdown of CARF resulted in an upregulation of the HDM2. This was supported by Western analysis of HDM2 in CARF overexpressing cells that showed reduced levels of expression of both exogenous (HDM2-YFP) and endogenous (HDM2) proteins (Fig. 3d). We also confirmed these findings by using HDM2 promoterdriven luciferase reporter in U2OS cells stably expressed with zinc inducible CARF expression vector. As shown in Fig. 3e, overexpression of CARF resulted in a reduced expression of HDM2 promoter-driven luciferase and it was abrogated by CARF silencing by siRNA. To get a final proof of transcriptional regulation of HDM2 by CARF, we performed RT-PCR analysis for HDM2 in CARF-compromised cells and detected a significant increase in HDM2 transcript level (Fig. 3f). These results strongly implied that CARF acts as a transcriptional repressor of HDM2 and may thus account for the ARFindependent effects of CARF on p53 function as observed in our earlier studies $(36,42)$.

We then examined if the transcriptional repressor function of CARF on HDM2 affects p53 function. As expected, CARF-knockdown cells showed higher amounts of HDM2 and lower levels of other p53 downstream proteins, such as, BAX, PUMA and p21WAF1 (Fig. 4a). The result was consistent with our earlier findings that the overexpression of CARF increases p53 function. Of note, consistent with our earlier studies, the level of p53 remained unchanged in CARFknockdown cells suggesting that an increase in HDM2 level due to depletion of CARF did not initiate degradation of p53. The decrease in the level of p53 downstream proteins, p21WAF1, PUMA and BAX therefore indicate that the transactivation function of $\mathrm{p} 53$ was decreased by a mechanism other than HDM2-mediated p53 degradation. To further support this observation, we employed a p53-dependent promoter-based reporter assay. As shown in Fig. 4b, p53dependent reporter activity was decreased, at least, 4-fold in CARF knockdown cells as compared to vector transfected control cells. These results demonstrated that an upregulation of HDM2 expression induced by knockdown of CARF modulated p53 functions. We earlier found that the expression of ARF and CARF are co-regulated; cells lacking ARF also lacked CARF (36). Our present findings on the degradation of CARF by HDM2 strongly imply that whereas CARF is degraded by HDM2, the presence of ARF in the same complex may stabilize it, possibly by nucleolar sequestration. In other words, the absence of ARF makes CARF susceptible to degradation by HDM2. Collectively, the study has demonstrated that CARF has potential to control the ARF-p53HDM2-p21WAF1 pathway at multiple points. Whereas an over-expression of CARF activates this pathway resulting in growth arrest, its simultaneous degradation by HDM2 (result of p53 activation) allows cells to enter cell cycle. To overcome this, CARF acts as a transcriptional repressor of HDM2 (Fig. 3). It was shown that HDM2 interacts with p21WAF1 and functions as its negative regulator independent of both its p53-regulatory and ubiquitination functions (45). Hence, the HDM2-repressor function of CARF may also affect p21WAF1, independent of p53. The only other protein known to interact with multiple members of the p53 pathway is Cyclin G1 that interacts with ARF, HDM2, and $\mathrm{p} 53$ in vitro and in vivo. Similar to CARF, it activates $\mathrm{p} 53$ function and is degraded by HDM2 through proteasome-mediated degradation (39).

CARF was first cloned as an ARF-binding protein that activated ARF-mediated p53 function $(36,40)$. In the absence of p14ARF, it was found to bind to p53 directly and resulted in its activation (42) suggesting that CARF activates the p53 pathway by its ARF-dependent and -independent p53 activation functions. Earlier studies also showed that the strong activation of $\mathrm{p} 53$ by CARF could be overcome, at least in part, by negative regulation of CARF by p53, possibly by degradation (42). In the present study, we showed that CARF directly binds to HDM2 (Fig. 1), becomes ubiquitylated in vivo and undergoes HDM2-mediated degradation (Fig. 2). The 
data explained the molecular mechanism of regulation of CARF by p53 and brought in focus another level of control that CARF puts on the p53 pathway. For the first time, we demonstrate a novel feedback loop in which CARF protects itself from HDM2-mediated degradation by acting as a transcriptional repressor of HDM2 (Fig. 3). In continuation with our earlier studies showing collaboration with ARF and p53 $(36,42)$, we demonstrated herein that CARF inactivates its own- and p53-antagonist, HDM2 and thus brings to light a novel regulatory loop of the CARF-p53-HDM2-p21WAF1 pathway. Collectively, CARF, by imposing multiple regulatory checkpoints, may act as a novel key regulator of p53 pathway.

\section{Acknowledgements}

This study was partly supported by grants from the NEDO (New Energy and Industrial Technology Development Organization) of Japan.

\section{References}

1. Hinds PW: The retinoblastoma tumor suppressor protein. Curr Opin Genet Dev 5: 79-83, 1995.

2. Kouzarides T: Functions of $\mathrm{pRb}$ and $\mathrm{p} 53$ : what's the connection? Trends Cell Biol 5: 448-450, 1995.

3. Vaziri $\mathrm{H}$ and Benchimol S: Alternative pathways for the extension of cellular life span: inactivation of $\mathrm{p} 53 / \mathrm{pRb}$ and expression of telomerase. Oncogene 18: 7676-7680, 1999.

4. Duncan EL, Wadhwa R and Kaul SC: Senescence and immortalization of human cells. Biogerontology 1: 103-121, 2000.

5. Zheleva DI, Lane DP and Fischer PM: The p53-Mdm2 pathway: targets for the development of new anticancer therapeutics. Mini Rev Med Chem 3: 257-270, 2003.

6. Brookes S, Rowe J, Gutierrez Del Arroyo A, Bond J and Peters G: Contribution of p16(INK4a) to replicative senescence of human fibroblasts. Exp Cell Res 298: 549-559, 2004.

7. Itahana K, Campisi J and Dimri GP: Mechanisms of cellular senescence in human and mouse cells. Biogerontology 5: 1-10, 2004.

8. Ito T, Nishida N, Fukuda Y, Nishimura T, Komeda T and Nakao K: Alteration of the p14(ARF) gene and p53 status in human hepatocellular carcinomas. J Gastroenterol 39: 355-361, 2004.

9. Larsen CJ: pRB, p53, p16INK4a, senescence and malignant transformation. Bull Cancer 91: 399-402, 2004.

10. Kamijo T, Zindy F, Roussel MF, Quelle DE, Downing JR, Ashmun RA, Grosveld G and Sherr CJ: Tumor suppression at the mouse INK4a locus mediated by the alternative reading frame product p19ARF. Cell 91: 649-659, 1997.

11. Quelle DE, Cheng M, Ashmun RA and Sherr CJ: Cancerassociated mutations at the INK4a locus cancel cell cycle arrest by p16INK4a but not by the alternative reading frame protein p19ARF. Proc Natl Acad Sci USA 94: 669-673, 1997.

12. Serrano M: The tumor suppressor protein p16INK4a. Exp Cell Res 237: 7-13, 1997.

13. Sharpless NE, Bardeesy N, Lee KH, Carrasco D, Castrillon DH, Aguirre AJ, Wu EA, Horner JW and DePinho RA: Loss of p16Ink4a with retention of p19Arf predisposes mice to tumorigenesis. Nature 413: 86-91, 2001.

14. Gibson SL, Dai CY, Lee HW, DePinho RA, Gee MS, Lee WM, Furth EE, Brensinger C and Enders GH: Inhibition of colon tumor progression and angiogenesis by the ink $4 \mathrm{a} /$ arf locus. Cancer Res 63: 742-746, 2003.

15. Lowe SW and Sherr CJ: Tumor suppression by Ink4a-Arf: progress and puzzles. Curr Opin Genet Dev 13: 77-83, 2003.

16. Hsieh JK, Chan FS, O'Connor DJ, Mittnacht S, Zhong S and $\mathrm{Lu} \mathrm{X}$ : RB regulates the stability and the apoptotic function of p53 via MDM2. Mol Cell 3: 181-193, 1999.

17. Yap DB, Hsieh JK, Chan FS and Lu X: Mdm2: a bridge over the two tumour suppressors, p53 and Rb. Oncogene 18: 7681-7689, 1999.
18. Weber JD, Kuo ML, Bothner B, Di Giammarino EL, Kriwacki RW, Roussel MF and Sherr CJ: Cooperative signals governing ARF-mdm2 interaction and nucleolar localization of the complex. Mol Cell Biol 20: 2517-2528, 2000.

19. Uchida C, Miwa S, Kitagawa K, Hattori T, Isobe T, Otani S, Oda T, Sugimura H, Kamijo T, Ookawa K, Yasuda $H$ and Kitagawa M: Enhanced Mdm2 activity inhibits pRB function via ubiquitin-dependent degradation. EMBO J 24: 160-169, 2004.

20. Stott FJ, Bates S, James MC, McConnell BB, Starborg M, Brookes S, Palmero I, Ryan K, Hara E, Vousden KH and Peters G: The alternative product from the human CDKN2A locus, p14(ARF), participates in a regulatory feedback loop with p53 and MDM2. EMBO J 17: 5001-5014, 1998.

21. Zhang Y, Xiong Y and Yarbrough WG: ARF promotes MDM2 degradation and stabilizes p53: ARF-INK4a locus deletion impairs both the Rb and p53 tumor suppression pathways. Cell 92: 725-734, 1998.

22. Honda R and Yasuda H: Association of p19(ARF) with Mdm2 inhibits ubiquitin ligase activity of $\mathrm{Mdm} 2$ for tumor suppressor p53. EMBO J 18: 22-27, 1999.

23. Weber JD, Jeffers JR, Rehg JE, Randle DH, Lozano G, Roussel MF, Sherr CJ and Zambetti GP: p53-independent functions of the p19 (ARF) tumor suppressor. Genes Dev 14: 2358-2365, 2000.

24. Zhang Y and Xiong Y: Control of p53 ubiquitination and nuclear export by MDM2 and ARF. Cell Growth Differ 12: 175-186, 2001.

25. Shmueli A and Oren M: Regulation of p53 by Mdm2: fate is in the numbers. Mol Cell 13: 4-5, 2004.

26. Vassilev LT: Small-molecule antagonists of p53-MDM2 binding: research tools and potential therapeutics. Cell Cycle 3: 419-421, 2004.

27. Vassilev LT, Vu BT, Graves B, Carvajal D, Podlaski F, Filipovic Z, Kong N, Kammlott U, Lukacs C, Klein C, Fotouhi N and Liu EA: In vivo activation of the $\mathrm{p} 53$ pathway by smallmolecule antagonists of MDM2. Science 303: 844-848, 2004.

28. Llanos S, Clark PA, Rowe J and Peters G: Stabilization of p53 by p14ARF without relocation of MDM2 to the nucleolus. Nat Cell Biol 3: 445-452, 2001.

29. Korgaonkar C, Zhao L, Modestou M and Quelle DE: ARF function does not require p53 stabilization or Mdm2 relocalization. Mol Cell Biol 22: 196-206, 2002.

30. Eymin B, Karayan L, Seite P, Brambilla C, Brambilla E, Larsen CJ and Gazzeri S: Human ARF binds E2F1 and inhibits its transcriptional activity. Oncogene 20: 1033-1041, 2001

31. Jackson MW, Lindstrom MS and Berberich SJ: MdmX binding to ARF affects Mdm2 protein stability and p53 transactivation. J Biol Chem 276: 25336-25341, 2001.

32. Karayan L, Riou JF, Seite P, Migeon J, Cantereau A and Larsen CJ: Human ARF protein interacts with Topoisomerase I and stimulates its activity. Oncogene 19: 836-848, 2001.

33. Martelli F, Hamilton T, Silver DP, Sharpless NE, Bardeesy N, Rokas M, DePinho RA, Livingston DM and Grossman SR: p19ARF targets certain E2F species for degradation. Proc Natl Acad Sci USA 98: 4455-4460, 2001.

34. Sugihara T, Kaul SC, Kato, J, Reddel RR, Nomura H and Wadhwa R: Pex19p dampens the p19ARF-p53-p21WAF1 tumor suppressor pathway. J Biol Chem 276: 18649-18652, 2001.

35. Vivo M, Calogero RA, Sansone F, Calabro V, Parisi T, Borrelli L, Saviozzi S and La Mantia G: The human tumor suppressor arf interacts with spinophilin/neurabin II, a type 1 proteinphosphatase-binding protein. J Biol Chem 276: 14161-14169, 2001.

36. Hasan MK, Yaguchi T, Sugihara T, Kumar PK, Taira K, Reddel RR, Kaul SC and Wadhwa R: CARF is a novel protein that cooperates with mouse p19ARF (human p14ARF) in activating p53. J Biol Chem 277: 37765-37770, 2002.

37. Menendez S, Khan Z, Coomber DG, Lane DP, Higgins M, Koufali MM and Lain S: Oligomerisation of the human ARF tumor suppressor and its response to oxidative stress. J Biol Chem 278: 18720-18729, 2003.

38. Rizos H, Diefenbach E, Badhwar P, Woodruff S, Becker TM, Rooney RJ and Kefford RF: Association of p14ARF with the p120E4F transcriptional repressor enhances cell cycle inhibition. J Biol Chem 278: 4981-4989, 2003. 
39. Zhao L, Samuels T, Winckler S, Korgaonkar C, Tompkins V, Horne MC and Quelle DE: Cyclin G1 has growth inhibitory activity linked to the ARF-Mdm2-p53 and pRb tumor suppressor pathways. Mol Cancer Res 1: 195-206, 2003.

40. Wadhwa R, Sugihara T, Hasan MK, Duncan EL, Taira K and Kaul SC: A novel putative collaborator of p19(ARF). Exp Gerontol 38: 245-252, 2003.

41. Sterian A, Kan T, Berki AT, Mori Y, Olaru A, Schulmann K, Sato F, Wang S, Paun B, Cai K, Hamilton JP, Abraham JM and Meltzer SJ: Mutational and LOH analyses of the chromosome 4q region in esophageal adenocarcinoma. Oncology 70: 168-172, 2006 .

42. Hasan MK, Yaguchi T, Minoda Y, Hirano T, Taira K, Wadhwa R and Kaul SC: Alternative reading frame protein (ARF)independent function of CARF (collaborator of ARF) involves its interactions with p53: evidence for a novel p53-activation pathway and its negative feedback control. Biochem J 380: 605-610, 2004.
43. Wadhwa R, Kaul SC, Miyagishi M and Taira K: Know-how of RNA interference and its applications in research and therapy. Mutat Res 567: 71-84, 2004

44. Wadhwa R, Yaguchi T, Kaur K, Suyama E, Kawasaki H, Taira K and Kaul SC: Use of a randomized hybrid ribozyme library for identification of genes involved in muscle differentiation. J Biol Chem 279: 51622-51629, 2004.

45. Zhang Z, Wang H, Li M, Agrawal S, Chen X and Zhang R: MDM2 is a negative regulator of $\mathrm{p} 21 \mathrm{WAF} 1 / \mathrm{CIP} 1$, independent of p53. J Biol Chem 279: 16000-16006, 2004. 